\title{
Text Analysis and Automatic Triage of Posts in a Mental Health Forum
}

\author{
Ehsaneddin Asgari ${ }^{1}$ and Soroush Nasiriany ${ }^{2}$ and Mohammad R.K. Mofrad ${ }^{1}$ \\ Departments of Bioengineering ${ }^{1}$ and Electrical Engineering and Computer Science ${ }^{2}$ \\ University of California, Berkeley \\ Berkeley, CA 94720, USA \\ asgari@ischool.berkeley.edu, snasiriany@berkeley.edu, mofrad@berkeley.edu
}

\begin{abstract}
We present an approach for automatic triage of message posts in ReachOut.com mental health forum, which was a shared task in the 2016 Computational Linguistics and Clinical Psychology (CLPsych). This effort is aimed at providing the trained moderators of ReachOut.com with a systematic triage of forum posts, enabling them to more efficiently support the young users aged 14-25 communicating with each other about their issues. We use different features and classifiers to predict the users' mental health states, marked as green, amber, red, and crisis. Our results show that random forests have significant success over our baseline mutli-class SVM classifier. In addition, we perform feature importance analysis to characterize key features in identification of the critical posts.
\end{abstract}

\section{Introduction}

Mental health issues profoundly impact the wellbeing of those afflicted and the safety of society as a whole (Üstün et al., 2004). Major effort is still needed to identify and aid those who are suffering from mental illness but doing so in a case by case basis is not practical and expensive (Mark et al., 2005). These limitations inspired us to develop an automated mechanism that can robustly classify the mental state of a person. The abundance of publicly available data allows us to access each person's record of comments and message posts online in an effor to predict and evaluate their mental health.

\subsection{Shared Task Description}

The CLPsych 2016 Task accumulates a selection of 65,514 posts from ReachOut.com, dedicated to providing a means for members aged 14-25 to express their thoughts in an anonymous environment. These posts have all been selected from the years 2012 through 2015. Of these posts, 947 have been carefully analyzed, and each assigned a label: green (the user shows no sign of mental health issues), amber (the user's posts should be reviewed further to identify any issues), red (there is a very high likelihood that the user has mental health issues), and crisis (the user needs immediate attention). These 947 postslabel pairs represent our train data. We then use the train data to produce a model that assigns a label to any generic post. A separate selection of 241 posts are dedicated as the test data, to be used to evaluate the accuracy of the model.

\section{Methods}

Our approach for automatic triage of posts in the mental health forum, much like any other classification pipeline, is composed of three phases: feature extraction, selection of learning algorithm, and validation and parameter tuning in a cross validation framework.

\subsection{Feature extraction}

Feature extraction is one of the key steps in any machine learning task, which can significantly influence the performance of learning algorithms (Bengio et al., 2013). In the feature extraction phase we extracted the following information from the given XML files of forum posts: author, the authors rank- 
ing in the forum, time of submission and editing, number of likes and views, the body of the post, the subject, the thread associated to the post, and changeability of the text. For the representation of textual data (subject and body) we use both tfidf and the word embedding representation of the data (Mikolov et al., 2013b; Mikolov et al., 2013a; Zhang et al., 2011). Skip-gram word embedding which is trained in the course of language modeling is shown to capture syntactic and semantic regularities in the data (Mikolov et al., 2013c; Mikolov et al., 2013a). For the purpose of training the word embeddings we use skip-gram neural networks (Mikolov et al., 2013a) on the collection of all the textual data (subject/text) of 65,514 posts provided in the shared task. In our word embedding training, we use the word2vec implementation of skip-gram (Mikolov et al., 2013b). We set the dimension of word vectors to 100 , and the window size to 10 and we sub-sample the frequent words by the ratio $\frac{1}{10^{3}}$. Subsequently, to encode a body/subject of a post we use tf-idf weighted sum of word-vectors in that post (Le and Mikolov, 2014). The features are summarized in Table 1 . To ensure being inclusive in finding important features, stop words are not removed.

\subsection{Automatic Triage}

The Random Forest (RF) classifier (Breiman, 2001) is employed to predict the users mental health states (green, red, amber, and crisis) from the posts in the ReachOut forum. A random forest is an ensemble method based on use of multiple decision trees (Breiman, 2001). Random forest classifiers have several advantages, including estimation of important features in the classification, efficiency when a large proportion of the data is missing, and efficiency when dealing with a large number of features (Cutler et al., 2012); therefore random forests fit our problem very well. The validation step is conducted over 947 labeled instances, in a 10xFold cross validation process. Different parameters of random forests, including the number of trees, the measure of split quality, the number of features in splits, and the maximum depth are tuned using cross-validation. In this work, we use Scikit implementation of Random Forests (Pedregosa et al., 2011).

Our results on the training set show that incorpo- ration of unlabeled data in the training using label propagation by means of nearest-neighbor search does not increase the classification accuracy. Therefore, the unlabeled data is not incorporated in the training.

For the comparison phase, we consider multiclass Support Vector Machine classifier (SVM) with radial basis function kernel as a baseline method (Cortes and Vapnik, 1995; Weston and Watkins, 1998).

\section{Results}

Our results show that random forests have significant success over SVM classifiers. The 4-ways classification accuracies are summarized in Table 3 . The evaluations on the test set for the random forest approach are summarized in Table 3.

\subsection{Important Features}

Random Forests can easily provide us with the most relevant features in the classification (Cutler et al., 2012; Breiman, 2001). Random Forest consists of a number of decision trees. In the training procedure, it can be calculated how much a feature decreases the weighted impurity in a tree. The impurity decrease for each feature can be averaged and normalized over all trees of the ensemble and the features can be ranked according to this measure (Breiman et al., 1984; Breiman, 2001). We extracted the most discriminative features in the automatic triage of the posts using mean decrease impurity for the best Random Forest we obtained in the cross-validation (Breiman et al., 1984).

Our results shows that from the top 100 features, $\frac{88}{100}$ were related to the frequency of particular words in the body of the post, $\frac{4}{100}$ were related to the posting/editing time (00:00 to 23:00) and the day in the month $\left(1^{\text {st }}\right.$ to $\left.31^{\text {th }}\right), \frac{4}{100}$ were indication of the author and author ranking, $\frac{2}{100}$ were related to the frequency of words in the subject, $\frac{1}{100}$ was the number of views, and $\frac{1}{100}$ was the number of likes a post gets.

The top 50 discriminative features, their importance, and their average values for each class are provided in Table 3.1. We have also presented the inverse document frequency (IDF) to identify how 


\begin{tabular}{|l||l|l|}
\hline \multicolumn{3}{|c|}{ Features Extracted from ReachOut forum posts } \\
\hline Feature & Description & Length \\
\hline Author & One hot representation of unique authors in 65755 posts. & 1605 \\
\hline Ranking of the author & One hot representation of the author category. & 25 \\
\hline Submission time & $\begin{array}{l}\text { Separated numerical representations of year, day, month, } \\
\text { and the hour that a post is submitted to the forum. }\end{array}$ & 4 \\
\hline Edit time & $\begin{array}{l}\text { Separated numerical representations of year, day, month, } \\
\text { and the hour that a post is edited in the forum. }\end{array}$ & 4 \\
\hline Likes & The number of likes a post gets. & 1 \\
\hline Views & The number of times a post is viewed by the forum users. & 1 \\
\hline Body & Tf-idf representation of the text in the body of the post. & 55758 \\
\hline Subject & Tf-idf representation of the text in the subject of the post. & 3690 \\
\hline Embedded-Body & $\begin{array}{l}\text { Embedding representation of the text in the body of the } \\
\text { post. }\end{array}$ & 100 \\
\hline Embedded-Subject & $\begin{array}{l}\text { Embedding representation of the text in the subject of the } \\
\text { post. }\end{array}$ & 100 \\
\hline Thread & One hot representation of the thread of the post. & 3910 \\
\hline Read only & If the post is readonly. & 1 \\
\hline
\end{tabular}

Table 1: List of features that have been used in the automatic triage of ReachOut forum posts

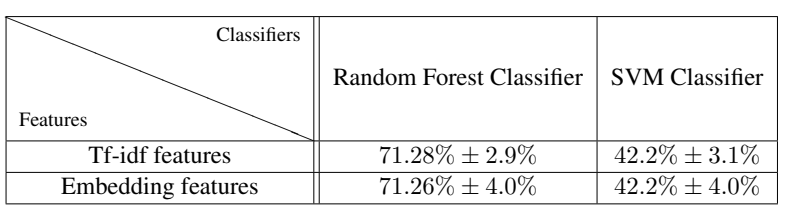

Table 2: The average 4-ways classification accuracies in 10xFold cross-validation for the random forest and support vector machine classifiers tuned for the best parameters on two different sets of features. Embedding features refer to use of embeddings for the body and the subject instead of tf-idf representations.

\begin{tabular}{|c||c|c|}
\hline Methods & Accuracy & Non-green vs . green accuracy \\
\hline Random Forest \& tf-idf features & $79 \%$ & $86 \%$ \\
\hline Random Forest \& embedding features & $78 \%$ & $86 \%$ \\
\hline
\end{tabular}

Table 3: The results of evaluation over 241 test data points.

much information each word has encoded within the collection of posts (Robertson, 2004). Many interesting patterns can be observed in the word usage of each class. For example, the word 'feel' significantly more often occurs in the red and crisis posts. Surprisingly, there were some stop-words among the most important features. For instance, words 'to' and 'not', on average occur in green posts $\frac{1}{2}$ of times of non-green posts. Another example is the usage of the word 'me', which occurs more frequently in non-green posts. Furthermore, the posts with more 'likes' are less likely to be non-green.

Subject: As indicated in Table 3.1 posts which have word 're' in their subjects are more likely to belong to the green class.

Time: As shown in Figure 1 and Table 3.1 the red posts on average are submitted on a day closer to the end of the month. In addition, the portion of red and crisis message posts in the interval of 5 A.M. to 7 A.M. was much higher than the green and amber posts.

\section{Conclusion}

In this work, we explored the automatic triage of message posts in a mental health forum. Using Random Forest classifiers we obtain a higher triage accuracy in comparison with our baseline method, i.e. a mutli-class support vector machine. Our results showed that incorporation of unlabeled data did not increase the classification accuracy of Random Forest, which could be due to the fact that Random Forests themselves are efficient enough in dealing with missing data points (Cutler et al., 2012). Furthermore, our results suggest that employing full vocabularies would be more discriminative than using sentence embedding. This could be interpreted as the importance of occurrence of particular words rather than particular concepts. In addition, taking advantage of the capability of Random Forest in the estimation of important features in classification, we explored the most relevant features contributing in the automatic triage.

\section{Acknowledgments}

Fruitful discussions with Meshkat Ahmadi, Mohsen Mahdavi, and Mohammad Soheilypour are gratefully acknowledged. 


\begin{tabular}{|c|c|c|c|c|c|c|c|}
\hline & & & & Green & Amber & Red & Crisis \\
\hline Rank & Feature & Importance & IDF & Average value & Average value & Average value & Average value \\
\hline 1 & body: you & 0.068 & 0.004 & $16.912 \pm 24.13$ & $3.941 \pm 10.238$ & $2.728 \pm 7.077$ & $2.432 \pm 8.605$ \\
\hline 2 & body: to & 0.059 & 0.012 & $4.948 \pm 5.739$ & $8.964 \pm 6.83$ & $9.408 \pm 7.265$ & $9.552 \pm 7.666$ \\
\hline 3 & subject: re & 0.053 & 0.03 & $3.904 \pm 1.871$ & $3.6 \pm 1.843$ & $3.246 \pm 2.637$ & $2.802 \pm 2.112$ \\
\hline 4 & \#oflikes & 0.027 & - & $0.749 \pm 1.104$ & $0.353 \pm 0.882$ & $0.155 \pm 0.453$ & $0.154 \pm 0.489$ \\
\hline 5 & body: just & 0.021 & 0.007 & $2.632 \pm 6.332$ & $6.69 \pm 8.962$ & $8.349 \pm 9.697$ & $8.702 \pm 9.992$ \\
\hline 6 & body: feeling & 0.02 & 0.009 & $0.884 \pm 3.463$ & $2.527 \pm 7.216$ & $4.227 \pm 9.606$ & $3.188 \pm 5.812$ \\
\hline 7 & body: don & 0.02 & 0.008 & $1.407 \pm 4.523$ & $3.998 \pm 7.302$ & $4.996 \pm 7.599$ & $9.074 \pm 13.873$ \\
\hline 8 & body: me & 0.019 & 0.006 & $2.73 \pm 6.471$ & $7.848 \pm 10.056$ & $9.321 \pm 11.432$ & $8.264 \pm 8.207$ \\
\hline 9 & \#ofviews & 0.016 & - & $96.016 \pm 53.53$ & $95.372 \pm 50.9$ & $92.158 \pm 53.715$ & $113.735 \pm 56.293$ \\
\hline 10 & body: know & 0.016 & 0.007 & $1.55 \pm 4.957$ & $3.976 \pm 7.806$ & $4.863 \pm 7.615$ & $8.218 \pm 11.262$ \\
\hline 11 & body: want & 0.015 & 0.008 & $0.548 \pm 2.587$ & $3.253 \pm 7.431$ & $3.875 \pm 8.172$ & $5.29 \pm 8.699$ \\
\hline 12 & body: anymore & 0.013 & 0.013 & $0.063 \pm 0.734$ & $0.523 \pm 2.578$ & $2.594 \pm 5.881$ & $4.709 \pm 9.327$ \\
\hline 13 & body: do & 0.013 & 0.007 & $1.987 \pm 5.58$ & $4.339 \pm 7.226$ & $4.741 \pm 7.275$ & $6.123 \pm 8.322$ \\
\hline 14 & body: and & 0.011 & 0.009 & $5.629 \pm 6.389$ & $7.953 \pm 7.687$ & $10.007 \pm 7.579$ & $6.749 \pm 5.905$ \\
\hline 15 & body: negative & 0.011 & 0.012 & $0.117 \pm 1.354$ & $1.184 \pm 4.354$ & $2.583 \pm 6.404$ & $4.446 \pm 8.769$ \\
\hline 16 & body: it & 0.01 & 0.007 & $6.89 \pm 9.562$ & $10.607 \pm 10.575$ & $9.079 \pm 9.527$ & $7.56 \pm 8.055$ \\
\hline 17 & post hour (1-24) & 0.01 & - & $9.922 \pm 4.325$ & $9.474 \pm 4.135$ & $9.118 \pm 4.585$ & $8.615 \pm 4.159$ \\
\hline 18 & body: my & 01 & 0.007 & $5.137 \pm 8.414$ & $9.722 \pm 10.703$ & $10.303 \pm 10.178$ & $7.928 \pm 10.775$ \\
\hline 19 & body: the & 01 & 0.011 & $4.744 \pm 5.5$ & $6.667 \pm 6.064$ & $5.95 \pm 5.578$ & $6.513 \pm 6.729$ \\
\hline 20 & body: for & 0.01 & 0.008 & $4.418 \pm 7.1$ & $3.894 \pm 5.61$ & $3.274 \pm 5.427$ & $6.135 \pm 5.89$ \\
\hline 21 & body: about & 0.009 & 0.008 & $1.646 \pm 4.452$ & $3.567 \pm 5.711$ & $2.11 \pm 4.567$ & $2.149 \pm 4.574$ \\
\hline 22 & body: so & 0.009 & 0.008 & $3.387 \pm 6.759$ & $4.95 \pm 7.102$ & $7.57 \pm 9.347$ & $5.02 \pm 7.942$ \\
\hline 23 & body: this & 0.009 & 0.008 & $2.624 \pm 5.609$ & $2.849 \pm 5.489$ & $5.302 \pm 5.768$ & $5.046 \pm 6.633$ \\
\hline 24 & post day (1-7) & 0.009 & - & $5.25 \pm 8.407$ & $15.719 \pm 8.625$ & $15.3 \pm 8.907$ & $17.436 \pm 8.217$ \\
\hline 25 & edit day (1-7) & 0.009 & - & $.25 \pm 8.407$ & $15.719 \pm 8.625$ & $15.3 \pm 8.907$ & $17.436 \pm 8.217$ \\
\hline 26 & body: can & 0.009 & 0.006 & $3.436 \pm 7.302$ & $4.297 \pm 6.909$ & $6.333 \pm 7.913$ & $12.029 \pm 12.095$ \\
\hline 27 & body: but & 0.008 & 0.006 & $3.588 \pm 6.988$ & $7.376 \pm 9.226$ & $5.354 \pm 7.634$ & $8.245 \pm 10.021$ \\
\hline 28 & body: not & 0.008 & 0.007 & $2.274 \pm 5.459$ & $5.037 \pm 8.02$ & $4.504 \pm 7.172$ & $3.901 \pm 6.398$ \\
\hline 29 & body: get & 0.008 & 0.006 & $1.672 \pm 4.627$ & $3.552 \pm 6.559$ & $4.505 \pm 8.02$ & $4.35 \pm 8.532$ \\
\hline 30 & edit hour (1-24) & 0.008 & - & $9.922 \pm 4.325$ & $9.474 \pm 4.135$ & $9.118 \pm 4.585$ & $8.615 \pm 4.159$ \\
\hline 31 & author $_{x}$ & 0.007 & - & $0.149 \pm 0.357$ & $0.072 \pm 0.259$ & $0.264 \pm 0.443$ & $0.308 \pm 0.468$ \\
\hline 32 & body: that & 0.007 & 0.007 & $4.244 \pm 7.687$ & $5.513 \pm 7.665$ & $4.905 \pm 7.357$ & $3.875 \pm 6.288$ \\
\hline 33 & body: of & 0.006 & 0.008 & $3.954 \pm 5.989$ & $4.902 \pm 6.235$ & $5.014 \pm 5.904$ & $5.425 \pm 6.389$ \\
\hline 34 & body: when & 0.005 & 0.008 & $1.689 \pm 4.25$ & $2.998 \pm 5.77$ & $2.779 \pm 5.249$ & $2.871 \pm 4.733$ \\
\hline 35 & body: even & 0.005 & 0.008 & $0.993 \pm 3.499$ & $1.513 \pm 4.099$ & $2.699 \pm 5.337$ & $4.37 \pm 8.633$ \\
\hline 36 & body: have & 0.005 & 0.005 & $4.081 \pm 7.854$ & $6.196 \pm 8.662$ & $6.415 \pm 8.511$ & $5.191 \pm 7.057$ \\
\hline 37 & body: cant & 0.005 & 0.013 & $0.033 \pm 0.764$ & $0.693 \pm 4.004$ & $1.589 \pm 4.911$ & $0.25 \pm 1.091$ \\
\hline 38 & body: all & 0.005 & 0.006 & $1.866 \pm 5.437$ & $3.487 \pm 6.37$ & $3.691 \pm 7.05$ & $2.804 \pm 6.987$ \\
\hline 39 & subject: into & 0.004 & 0.187 & $0.099 \pm$ & $0.391 \pm 0.941$ & $0.838 \pm 1.249$ & $0.728 \pm 1.201$ \\
\hline 40 & body: what & 0.004 & 0.008 & $1.813 \pm 4.463$ & $2.725 \pm 4.901$ & $2.778 \pm 4.744$ & $2.577 \pm 5.045$ \\
\hline 41 & ody: everything & 0.004 & 0.01 & $0.262 \pm 1.903$ & $0.64 \pm 2.8$ & $1.726 \pm 4.957$ & $1.376 \pm 3.576$ \\
\hline 42 & body: usernam & 0.004 & 0.016 & $1.096 \pm 4.881$ & $1.394 \pm 5.164$ & $0.938 \pm 3.523$ & $1.608 \pm 4.565$ \\
\hline 43 & body: in & 0.004 & 0.009 & $3.467 \pm 6.878$ & $3.311 \pm 4.559$ & $4.241 \pm 5.246$ & $3.175 \pm 4.247$ \\
\hline 44 & body: feel & 0.004 & 0.007 & $1.477 \pm 4.989$ & $3.145 \pm 6.323$ & $5.187 \pm 8.689$ & $3.746 \pm 6.598$ \\
\hline 45 & body: try & 0.004 & 0.009 & $0.683 \pm 3.816$ & $1.465 \pm 4.957$ & $1.46 \pm 3.793$ & $1.902 \pm 4.574$ \\
\hline 46 & body: anything & 0.004 & 0.007 & $0.541 \pm 3$ & $1.602 \pm 4.745$ & $2.195 \pm 5.751$ & $4.237 \pm 10.067$ \\
\hline 47 & body: am & 0.004 & 0.008 & $1.162 \pm 5.241$ & $1.655 \pm 4.619$ & $2.523 \pm 5.922$ & $1.642 \pm 4.584$ \\
\hline 48 & body: at & 0.004 & 0.007 & $2.033 \pm 5.47$ & $3.349 \pm 6.469$ & $3.661 \pm 6.051$ & $4.058 \pm 6.735$ \\
\hline 49 & body: with & 0.004 & 0.01 & $2.029 \pm 4.01$ & $3.189 \pm 5.024$ & $2.679 \pm 3.776$ & $1.591 \pm 2.872$ \\
\hline 50 & body: safe & 0.004 & 0.012 & $0.342 \pm 2.802$ & $0.163 \pm 1.801$ & $0.662 \pm 3.339$ & $2.907 \pm 6.549$ \\
\hline
\end{tabular}

Table 4: The 50 most discriminative features of posts and their mean values for each class of green, amber, red, and crisis, which are ranked according to their feature importance. For the words we have also provided their IDF.
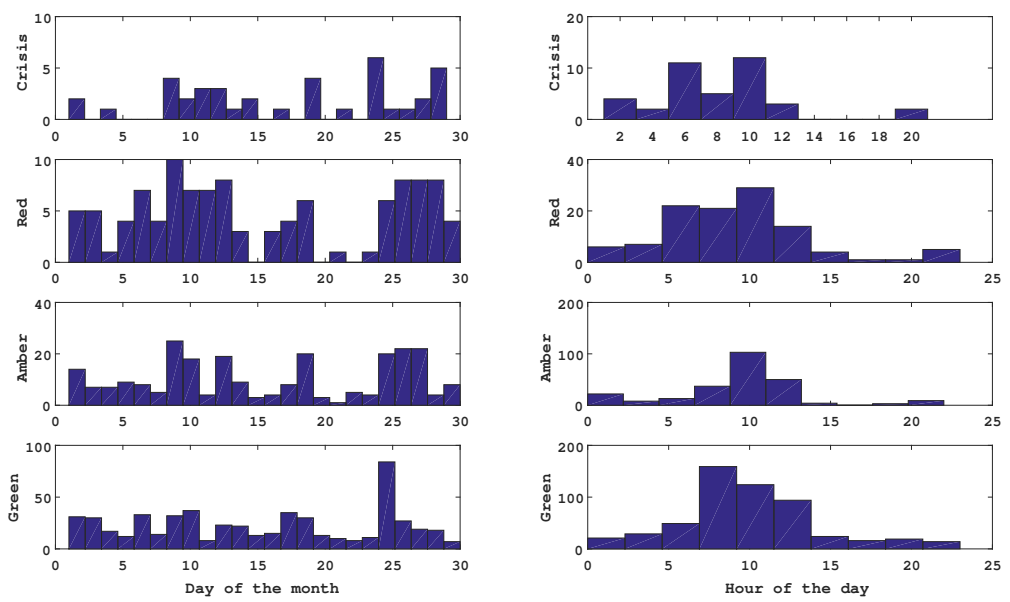

Figure 1: Histogram of message posting time distribution for each mental health state (crisis, red, amber, and green). The left plots show distribution of posts in days of the month (1-31) and the right plots show the distribution of the hours of the day. 


\section{References}

Yoshua Bengio, Aaron Courville, and Pierre Vincent. 2013. Representation learning: A review and new perspectives. Pattern Analysis and Machine Intelligence, IEEE Transactions on, 35(8):1798-1828.

Leo Breiman, Jerome Friedman, Charles J Stone, and Richard A Olshen. 1984. Classification and regression trees. CRC press.

Leo Breiman. 2001. Random forests. Machine learning, 45(1):5-32.

Corinna Cortes and Vladimir Vapnik. 1995. Supportvector networks. Machine learning, 20(3):273-297.

Adele Cutler, D Richard Cutler, and John R Stevens. 2012. Random forests. In Ensemble Machine Learning, pages 157-175. Springer.

Quoc V Le and Tomas Mikolov. 2014. Distributed representations of sentences and documents. arXiv preprint arXiv:1405.4053.

Tami L Mark, Rosanna M Coffey, Rita Vandivort-Warren, Hendrick J Harwood, et al. 2005. Us spending for mental health and substance abuse treatment, 19912001. Health Affairs, 24:W5.

Tomas Mikolov, Kai Chen, Greg Corrado, and Jeffrey Dean. 2013a. Efficient estimation of word representations in vector space. arXiv preprint arXiv:1301.3781.

Tomas Mikolov, Ilya Sutskever, Kai Chen, Greg S Corrado, and Jeff Dean. 2013b. Distributed representations of words and phrases and their compositionality. In Advances in neural information processing systems, pages 3111-3119.

Tomas Mikolov, Wen-tau Yih, and Geoffrey Zweig. 2013c. Linguistic regularities in continuous space word representations. In HLT-NAACL, pages 746751.

Fabian Pedregosa, Gaël Varoquaux, Alexandre Gramfort, Vincent Michel, Bertrand Thirion, Olivier Grisel, Mathieu Blondel, Peter Prettenhofer, Ron Weiss, Vincent Dubourg, et al. 2011. Scikit-learn: Machine learning in python. The Journal of Machine Learning Research, 12:2825-2830.

Stephen Robertson. 2004. Understanding inverse document frequency: on theoretical arguments for idf. Journal of documentation, 60(5):503-520.

TB Üstün, Joseph L Ayuso-Mateos, Somnath Chatterji, Colin Mathers, and Christopher JL Murray. 2004. Global burden of depressive disorders in the year 2000. The British journal of psychiatry, 184(5):386-392.

Jason Weston and Chris Watkins. 1998. Multi-class support vector machines. Technical report, Citeseer.

Wen Zhang, Taketoshi Yoshida, and Xijin Tang. 2011. A comparative study of $\mathrm{tf}^{*} \mathrm{idf}$, lsi and multi-words for text classification. Expert Systems with Applications, 38(3):2758-2765. 